\title{
GIUDICATI E SIGNORIE. DUE PERCORSI DI POTERE NEL MEDIOEVO A CONFRONTO ${ }^{1}$
}

\author{
"GIUDICATI" AND SEIGNIORIES. \\ COMPARISON OF TWO WAYS TOWARDS POWER IN THE MIDDLE AGES
}

\author{
LUCA DEMONTIS \\ Università di Milano
}

\begin{abstract}
Riasunto: Nel XIII secolo entra in crisi l'istituzione dell'Impero: il potere politico di cui esso è depositario si frantuma in poteri limitati e particolari. In questo lavoro voglio mettere a confronto due percorsi di potere locale: da una parte la signoria della famiglia della Torre a Milano, una delle prime in Italia, punto di arrivo della lotta contro l'Impero per le libertà comunali; dall'altra i giudicati della Sardegna, istituzione derivante dalla dissoluzione dell'Impero Bizantino nell'isola. Di tutti e due esamino le modalità dell'acquisizione del potere, il contesto in cui hanno operato, il rapporto tra città e contado, la funzione del "popolo" nell'attribuzione del potere, la necessità di una legittimazione da parte dell'autorità imperiale.
\end{abstract}

Parole chiave: Signoria; Giudicati; Potere; Impero; Chiesa; Città e contado.

\begin{abstract}
In the 13th century the Empire as political institution was in a state of crisis. The Holy Roman German Empire and the Byzantine Empire were reshaped by extensive fragmentation into regional districts with political autonomy, resulting in a more limited influence for both emperors. Here, I have compared two different paths toward local power, the "Seigniory" and the "Giudicati". The Seigniory of the family "della Torre" over Milan was one of the first in Italy and is a paradigm of acquisition of political autonomy within the Holy Roman German Empire. Conversely, the "Giudicati" were local kingdoms that arose in Sardinia from the decay of the Byzantine Empire. I discuss the ways by which these regional political institutions arose, their recognition by the emperor, the context in which they acted, the relations between city and peasantry, the role of the "people" in awarding the power.
\end{abstract}

Keywords: Seigniory; Giudicati; Power; Empire; Church; City and peasantry.

${ }^{1}$ Le principali abbreviazioni di cui faccio uso nel presente articolo sono: Francesco Cesare CASUla, Dizionario Storico Sardo, Sassari, 2001 = CASULA, Dizionario; A. SOLMI, Studi storici sulle istituzioni della Sardegna nel Medio Evo, Cagliari, $1917=$ SolmI, Studi; G. GIULINI, Memorie spettanti alla storia, al governo ed alla descrizione della città e della campagna di Milano nel secoli bassi, IV, Milano, 1854-18572 = GIULINI, Memorie. 


\section{SOMMARIO}

1. Introduzione. 2. La signoria dei della Torre (Torriani) di Milano e l'espansione in "Lombardia". 3. Dall'Impero di Bisanzio ai giudicati: i giudici d'Arborea e l'espansione in Sardegna. 4. Conclusioni.

\section{INTRODUZIONE}

Queste riflessioni si riferiscono a due ambiti geografici: la Sardegna, dove si sviluppano i giudicati dopo l'abbandono dell'isola da parte dell'Impero d'Oriente, e la penisola italiana, in particolare Milano, teatro delle prime sperimentazioni del potere signorile.

Dopo un breve quadro generale del secolo XIII, cercherò di fare un confronto tra due realtà: la signoria della famiglia della Torre (Torriani) a Milano e i giudicati della Sardegna, in particolare quello di Arborea.

Ognuna di queste realtà verrà vista nel suo percorso di avvicinamento al potere, quando si verifica il venir meno dell'autorità imperiale. Mentre in Sardegna la transizione dal potere imperiale a quello giudicale avviene quasi per evoluzione naturale, nell'Italia centro-settentrionale, e in particolare a Milano, il potere passa attraverso lotte militari e politiche violente dall' Impero ai comuni, e da questi alla signoria.

Giudicati e signorie arrivano quindi alla gestione del potere con un percorso assai differente.

In questo articolo si toccano diverse problematiche: la legittimazione del potere e il suo riconoscimento da parte di un'autorità esterna; il passaggio dell'isola di Sardegna dall'orbita dell'Impero d'Oriente all'ambito occidentale; il rapporto tra città e contado in Lombardia e in Sardegna; la funzione del Papato e dell'Impero in relazione ai nuovi centri di potere emergenti.

Durante il XIII secolo avvengono importanti cambiamenti nelle istituzioni politiche: l'Impero e il Papato sono in crisi e non sanno adattarsi alle nuove esigenze.

L'Impero perde nel 1250 Federico II. Nonostante la sua saggezza politica non era riuscito a elaborare vie nuove nei rapporti coi comuni: seguendo la stessa politica del nonno, aveva scelto di combatterli per riaffermare l'autorità imperiale. Ma ormai, inevitabilmente, l'unico centro del potere politico universalmente e teoricamente riconosciuto, l'Impero, si stava frantumando in una serie di poteri limitati e particolari ${ }^{2}$. La decadenza dell'Impero quindi aveva delle cause profonde che andavano oltre le sconfitte militari di Manfredi e Corradino.

\footnotetext{
${ }^{2} \mathrm{Si}$ tratta di una "pluralizzazione di poteri e non di dissoluzione del potere" (Giuseppe SERGI, L'idea di Medioevo, in AA.VV., Storia medievale, Roma, 1998, p. 39.
} 
In Germania esso è caratterizzato dallo strapotere dei grandi feudatari, specie dei principi elettori, e dal lungo periodo di interregno ${ }^{3}$.

In Francia, dopo la sconfitta degli anglo-imperiali a Bouvines (1214), il potere regio si rafforza.

In Italia l'affermazione dell'autonomia comunale sottrae al controllo imperiale molte città del centro e del nord della penisola. Nuovi ceti sociali emergenti nei liberi comuni sperimentano forme di governo innovative, non ancora teorizzate, spinti da necessità contingenti. Propongono un potere che viene dal basso e che deve trovare le sue motivazioni non più nell'autorevolezza delle teorie sino allora conosciute, ma nella prassi.

Con l'elezione di Rodolfo d'Asburgo e la fine dell'interregno, si verificano importanti cambiamenti nella politica imperiale: gli imperatori decideranno, per esempio, di non intervenire più in Italia direttamente, come facevano i loro predecessori ${ }^{4}$.

L'autorità imperiale, dalla quale i poteri locali cercano il distacco e l'indipendenza, verrà tuttavia riconosciuta come fonte di legittimazione formale di questi stessi poteri già affermati, ma senza stabilire un rapporto di dipendenza tra le parti. A un signore che voglia legittimare il proprio potere basta che ottenga dall'imperatore, a titolo oneroso, pagando grosse somme, o schierandosi nel partito imperiale, il titolo altisonante, ma dagli incerti attributi, di vicario imperiale: esso viene attribuito ad personam, non è trasmissibile in eredità e quindi è limitato nel tempo.

La Sede Apostolica, da parte sua, deve fronteggiare non solo i nemici esterni che si sono coalizzati nel movimento ghibellino, ma anche la preoccupante espansione dei movimenti ereticali pericolosamente centrifughi.

In campo politico il papa assume il comportamento disinvolto di un principe temporale: si appoggia agli Angioini per combattere gli eredi di Federico II e i loro sostenitori ghibellini, ma una volta cessata la minaccia sveva e rivelatasi oppressiva l'alleanza con Carlo d'Angiò ${ }^{5}$, rigetta quest'ulti-

${ }^{3}$ Iniziato nel 1254, durò vent'anni e fu caratterizzato dalle lotte per la successione. Nel 1257 furono eletti contemporaneamente al trono di re dei Romani Alfonso X di Castiglia el Sabio, sostenuto dall'arcivescovo di Treviri, e Ricçardo di Cornovaglia, incoronato in Aquisgrana dall'arcivescovo di Colonia. La Sede Apostolica, in un primo momento favorevole ad Alfonso, si era poi orientata verso Riccardo, quando il re di Castiglia era divenuto capo del ghibellinismo internazionale. Morto Riccardo, fu eletto dal Kurkollegıum a Francoforte Rodolfo d'Asburgo il 29 settembre 1273, col gradimento della curia romana, che aveva convinto Alfonso a rinunciare al titolo (Antonio PADOA SCHIOPPA, Il diritto nella storia d'Europa - Il medioevo, I, Padova, 1995 , p. 241).

${ }^{4}$ Tutt'al più verranno solo per farsi coronare a Roma, deludendo, come nel caso di Enrico VII, quanti avevano invocato una sua discesa in Italia. Oppure, senza successo, per recuperare il controllo dell'Impero sull'Italia, come Carlo IV. A lui si deve la Bolla d'oro del 1356 che stabiliva le regole definitive per l'elezione dell'imperatore, riservandola ai sette grandi elettori ed escludendo l'ingerenza del papato.

${ }^{5} \mathrm{La}$ filiale protezione del "diletto figlio" non era meno esigente della "aborrita tirannịa dell'alunno di Satana” Manfredi (G. FRANCESCHINI, La vita sociale e politica nel Duecento, in Storia di Milano Treccani, IV, Milano, 1954, p. 323). 
mo e prende accordi col neo-eletto imperatore Rodolfo d'Asburgo. Per la sua azione politica la Sede Apostolica fa largo uso di concessione di feudi e del titolo di vicario apostolico ${ }^{6}$ e non esita a fare uso della scomunica e dell'interdetto.

Per contrastare i movimenti ereticali e per riportare le masse popolari alla Chiesa si era rivelata più efficace l'azione dei nuovi ordini religiosi dei Domenicani e dei Minori Francescani ${ }^{7}$.

E in questa fase di crisi dell'Impero e del Papato che si incontrano tra loro due esigenze, una di domanda e l'altra di offerta: da una parte l'imperatore e il papa, le due supreme autorità, ricercano consensi e sostenitori; dall'altra i nuovi detentori del potere, le signorie, ritengono che il potere proveniente dal basso, ottenuto col favore del popolo, debba avere un riconoscimento ufficiale dall'alto, cioè dall'autorità tradizionale.

Alcune famiglie della penisola, di cui si vedrà un esempio più avanti, subito dopo il 1250 hanno un potere già consolidato di tipo signorile in una città e nel suo territorio, $\mathrm{o}$ in ambito anche più vasto, ma non lo sentono né sicuro né stabile, giacché si basa solo sul consenso popolare, per sua natura aleatorio.

Proprio per conferirgli maggiore stabilità e sicurezza, e per farlo riconoscere a livello internazionale, pensano di legittimarlo ricorrendo a un'autorità superiore, ritornando così a una concezione del potere di tipo feudale. Il marchese Uberto Pelavicino ${ }^{8}$, per esempio, si era attribuito nel 1254 il titolo di "vicario generale dell'Impero in Lombardia"9. Così i marchesi d'Este, già affermati nella loro signoria, ottengono il titolo di vicari apostolici nella città di Ferrara ${ }^{10}$. Nel 1267, quando in Italia si afferma l'autorità di Carlo d'Angiò, Goffredo della Torre, esponente della famiglia al potere in Milano, è nominato vicario angioino a Firenze.

Questa indagine riguarda i percorsi verso il potere signorile di due famiglie in contesti che, sebbene geograficamente vicini tra loro, appaiono

${ }^{6}$ Durante tutto il secolo XIII c'erano addirittura dei regni che per un certo periodo erano stati vassalli della Sede Apostolica, come il regno d'Inghilterra, il regno d'A Aragona e il regno normanno-svevo di Sicilia. Del Regnum Sardiniae et Corsicae si parlerà più avanti.

${ }^{7}$ August Franzen, Breve storia della Chiesa, Brescia, 1987, p. 218.

${ }^{8}$ Da una parte agiva con la forza delle armi e con la diplomazia per attirare nella sua orbita il maggior numero di comuni della Lombardia, dall'altra agiva sempre secondo i principi del dominio feudale, ottenuto o per eredità paterna o dall'imperatore, pensando che questo potere così espresso fosse più sicuro di quello ottenuto con fatica nei liberi comuni. Questo personaggio è portato dal Sestan come esempio del nesso che lega il feudalesimo superstite con la nascita della signoria (Ernesto SESTAN, Le origini delle signorie cittadine: un problema storico esaurito?, in La crisi degli ordinamenti comunali e le origini dello stato del Rinascimento, a c. di Giorgio CHITTOLINi, Bologna, 1979, pp. 68 sgg.).

${ }^{9}$ Giancarlo ANDENNA, Il concetto geografico-politico di Lombardia nel Medioevo, in Storia della Lombardia 1. Dalle origini al Seicento, a c. di Livio ANTONIELLI e Giorgio CHITTOLINI, Bari, 2003, p. 96.

${ }^{10}$ E. SESTAN, Le origini delle signorie, cit., p. 66. 
assai diversi per le caratteristiche cronologiche, politiche e culturali che li contraddistinguono. Non si tratta solo di raccontare la storia di due casati che al culmine del successo si trovano alla guida di un'entità statale regionale, ma anche di esaminare quali circostanze abbiano favorito la loro ascesa, quale sia stato il rapporto di ognuna di loro col centro del potere politico (Impero) e con le periferie del rispettivo potere regionale.

Di riflesso, dietro le quinte, si intravede quindi anche un profilo delle due entità che si definiscono "Impero Romano", quello "Sacro" e "Germanico" in Occidente e quello "Bizantino" in Oriente, e il modo con cui ognuno di essi esercita il potere.

Si può vedere anche la politica del papato sia in campo religioso che in campo politico: nella penisola guida il movimento antighibellino; per quanto concerne la Sardegna, isola legata per un certo tempo all'Impero Bizantino, esso è interessato a riportarla in ambito occidentale e poi a gestirla con intervento diretto, vantando il diritto di disporne come feudo.

Dopo questa introduzione, nelle due parti che seguono esporrò per grandi linee la storia della famiglia della Torre, una delle prime nella penisola ad esercitare un potere di tipo signorile, anche se con caratteristiche particolari, e la storia dei giudicati, quello d'Arborea in particolare. Cercherò di mettere a confronto le istituzioni sorte dalle rovine dell'Impero di Bisanzio con le ultime esperienze che concludono la vita dei comuni italiani.

\section{LA SIGNORIA DEI DELlA TORRE (TORRIANI) DI MILANO E L'ESPANSIONE IN “LOMBARDIA”}

Alcune signorie che nascono nel XIII secolo si affermano con un processo lento, mascherato da "sembianze di continuità", nel rispetto formale degli istituti cittadini, i consigli e le assemblee, "che vennero però svuotati di fatto del loro significato, privati di competenze e facoltà decisionali" ${ }^{11}$.

È il caso della famiglia della Torre a Milano. Originaria della Valsassina, di cui aveva l'investitura comitale dall'arcivescovo di Milano ${ }^{12}$, si stabilì durevolmente nella città ambrosiana a partire dal 1240. Alcuni suoi membri vi avevano già ricoperto incarichi consolari ${ }^{13}$, ma saranno gli avvenimenti del 1237 a decidere l'affermazione di questa famiglia all'interno delle istituzioni comunali.

\footnotetext{
${ }^{11}$ Elisa OCCHIPINTI, L'Italia dei comuni. Secoli XI-XIII, Roma, 2000, p. 92.

${ }^{12}$ Gian Piero BognetTI, Le miniere della Valtorta e $i$ diritti degli arcivescovi di Milano, in "Archivio Storico Lombardo", anno LIII (1926), p. 281.

${ }^{13}$ Come testimoniano Gli atti del comune di Milano fino all'anno MCCXVI, a c. di Cesare MANARESI, e altri documenti dell'Archivio di Stato di Milano, Fondo Pergamene.
} 
Nella battaglia di Cortenuova contro Federico II i Milanesi persero 5000 uomini e il podestà Pietro Tiepolo. Il resto dell'esercito in rotta fu assalito dai Bergamaschi, prima neutrali e poi alleati dell'imperatore. In quell'occasione Pagano della Torre soccorse con i suoi armati i Milanesi superstiti, proteggendoli, curandoli e rimandandoli a Milano a sue spese ${ }^{14}$. Nel 1240, non dimentico di tale gesto, il "popolo" milanese chiamò Pagano "per capitano e difensore col titolo di podestà" 15 .

Accettando la carica, Pagano e i della Torre si schierano a favore della pars populi nella lunga controversia contro la pars nobilium, ${ }^{16}$ e la loro fortuna, ossia la marcia al potere, andrà sempre crescendo finché il potere resterà nelle mani del solo "popolo" 17 . Occorre brevemente chiarire che nel "popolo" o pars populi di Milano confluiscono non soltanto gli strati più modesti della popolazione cittadina, lavoratori e artigiani, che costituiscono la "Credenza di S. Ambrogio", ma anche mercanti facoltosi, molti notai e giudici, che si riconoscono nella "Motta". Chi veniva eletto come guida non apparteneva alle categorie più basse ma, come nel caso di Pagano della Torre, a una famiglia nobile, che si impegnava a rappresentare la volontà popolare.

Nel nostro caso la politica del "popolo" al governo del comune aveva un duplice obiettivo: la lotta ai privilegi signorili, detenuti ancora da numerose famiglie e da alcuni enti ecclesiastici, proprietari di grandi patrimoni immobiliari e di castelli, e l'espansione della città verso il contado, in modo da controllarlo e organizzarne la produzione in funzione del mercato cittadino.

Poiché il governo comunale voleva tassare il clero e non lottava abbastanza contro gli eretici, la Sede Apostolica intervenne in modo duro sull'operato dell'amministrazione di Milano inviandovi come legato Gregorio da Montelongo, che assunse, assieme all'arcivescovo Leone da Perego, funzioni di governo nel 1240-1241.

La nomina papale nel 1241 di Leone da Perego ${ }^{18}$ dei frati Minori ad arcivescovo di Milano, la seconda sede metropolitica per importanza della cristianità occidentale, è in linea con la politica della Sede Apostolica di appoggiarsi ai nuovi ordini mendicanti sia nella difesa della libertas ecclesie sia nella lotta contro l'eresia ${ }^{19}$ e contro il ghibellinismo. Questa nomina, segno

\footnotetext{
${ }^{14}$ L'episodio è riportato in Bernardino CoRIO, Storia di Milano, a c. di Anna MorISI GuERRA, Torino, 1978, p. 76; Galvano FIAMMA, Manipulus Florum sive istoria Mediolanensis, in Ludovico Antonio MURATORI, Rerum Italicarum Scriptores, XI, Mediolani, 1727, col. 673.

${ }^{15}$ Non tutto il casato dei della Torre si trasferì a Milano.

${ }^{16}$ Giovanni TABACCO, Egemonie sociali e strutture del potere nel Medioevo italiano, Torino, $2000^{3}$, pp. 291-292.

${ }^{17}$ Sul significato del termine cfr. John KoENIG, Il "popolo" dell'Italia del Nord nel XIII secolo, Bologna, 1986.

${ }^{18}$ Proclamato beato dalla Chiesa.

${ }^{19} \mathrm{~A}$ Bonaventura da Bagnoregio, alla guida dell'ordine, sarebbe spettato in quello stesso periodo anche il compito di sradicare dall'ordine la dissidenza gioachimita (Anna BENVENUTI, La religiosità eterodossa, in AA. VV., Storia Medievale, Roma, 1998, p. 528).
} 
della stima del papa, che affiderà ai Minori delicati incarichi, segna l'ingresso dell'ordine nelle cariche ecclesiastiche e in seguito anche nelle università e nella cultura dotta del tempo ${ }^{20}$.

Secondo alcuni studiosi l'esperienza di Leone da Perego al governo di Milano fu il primo esperimento di signoria ${ }^{21}$. La curia di Roma così poteva controllare da vicino i vescovi nella difesa della libertas ecclesie, per evitare compromessi e cedimenti ${ }^{22}$. Essa vuole inoltre che la lotta all'eresia sia portata avanti non solo con metodi repressivi organizzati dall'Inquisizione, ma anche con una intensa predicazione affidata ai nuovi ordini religiosi, e con il coinvolgimento dei laici nella vita della Chiesa.

I della Torre, che impongono con successo le tasse agli ordini religiosi e agli altri enti ecclesiastici, a nome delle istituzioni cittadine si impegnano anche in un altro duro compito: combattere $i$ "nobili" in esilio, che vagano nel contado alla ricerca di appoggi da parte dei rustici e di alleanze coi "nobili" fuorusciti di città confinanti.

Ma le lotte armate tra le fazioni interne mettono in pericolo la coesione del comune come organismo statale: una grave crisi sociale e politica mina la sua autonomia, faticosamente conquistata combattendo contro nemici esterni. Le forze centrifughe (signorie rurali, borghi, castelli), che il comune aveva cercato di assorbire sotto la sua giurisdizione, riprendono vigore e cercano di sottrarsi al controllo delle autorità cittadine.

Questa crisi facilita un'evoluzione verso un'organizzazione più stabile del potere e porta a creare "ordinamenti territoriali più esattamente definiti e strutturati" 23 che diano garanzia di maggior durata. Uno dei frutti di questa evoluzione è la trasformazione degli ordinamenti repubblicani in forme monocratiche e tiranniche ${ }^{24}$.

Quando nel comune la tensione interna supera la soglia di rottura, e questo avviene quando muore Federico II, principale nemico esterno contro cui erano state convogliate tutte le energie, c'è un'unica via d'uscita obbligata:

${ }^{20}$ Il francescano Guglielmo di Rubruk tra il 1252 e il 1255 percorre la Mongolia recando lettere di Luigi IX di Francia. Il resoconto del viaggio, 1' Itinerarium, precede di molti anni il più famoso Milione di Marco Polo (M. OLDANI, Culture: dotta, popolare, orale, in AA. VV. Storia medievale, cit., p. 403).

${ }^{21} \mathrm{Cfr}$. Roberto PERELli CIPPO, Tra arcivescovo e comune. Momenti e personaggi del Medioevo Milanese, Milano, 1995.

${ }^{22}$ Il papa nọ nominerà arcivescovo di Milano Raimondo della Torre perché i legami familiari potevano impedirgli di resistere alle decisioni del comuni contrarie agfi interessi della Chiesa.

${ }^{23}$ G. ChitTolini, Introduzione, in La crisi degli ordinamenti comunali, cit., p. 10.

${ }^{24}$ E. OCCHIPINTI, L'Italia dei comuni, cit., p. 92. 
creare un apparato di governo più stabile, che resista alle tendenze disgregatrici, ossia un regime statale vero e proprio ${ }^{25}$.

Conseguenza dei nuovi ordinamenti statali è la separazione dell'esercizio del potere "da ogni concorso di gruppi mutevoli per consistenza e per interessi rappresentati", la creazione di strutture politiche e ordinamenti militari staccati dal corpo sociale e al di sopra di esso ${ }^{26}$.

Nessuno dei nuovi signori cittadini, di grandi città come Milano o di centri più modesti, anche se è arrivato al potere col sostegno della pars populi, si comporta da "borghese autentico"; tutti sono "con un piede, se non con due ben immersi nella superstite società feudale", sia perché provengono da quel mondo di cui hanno conservato lo spirito, sia perché possono contare su vaste clientele armate rurali e cittadine e su castelli, sia, infine, perché dispongono di molti legami esterni e hanno le capacità di mettersi a capo di "partes intercittadine" 27.

Nella pianura Padana in particolare la storia dei singoli comuni diventa una storia di sistemi di relazione e di alleanze: un comune poteva sopravvivere solo se legato a forze egemoni da accordi politici e militari, anche col rischio di dover accettare un vincolo stabile di dipendenza politica ${ }^{28}$.

Finché i della Torre occupano cariche istituzionali del comune di Milano, per esempio quella di podestà, pur essendo sostenuti dalla pars populi che essi difendono contro i potenti, devono in qualche modo rendere conto del loro operato e mediare per conciliare i due partiti: nel 1258, il 4 aprile, dopo innumerevoli episodi di violenze reciproche, si approvarono solennemente i "capitoli della concordia" ${ }^{29}$ che sancivano la pace tra la pars nobilium e la pars populi: conseguentemente tutti i bandi inflitti dal comune e i provvedimenti punitivi adottati durante i disordini venivano aboliti, gli esuli potevano rientrare in città e venivano restituiti loro $i$ beni ${ }^{30}$.

Per quanto riguarda l'aspetto istituzionale si stabilì che tutte le magistrature, gli uffici, le cariche e persino i trombettieri dovessero essere scelti egualmente tra le due parti ${ }^{31}$.

\footnotetext{
${ }^{25}$ Giovanni TABACCO, La storia politica e sociale, in Storia d'Italia, 2, t. I, Dalla caduta dell'Impero Romano al secolo XVIII, a c. di Ruggiero ROMANO e Corrado VIVANTI, Torino, 1974 , pp. 240, 257.

${ }^{26} \mathrm{G}$. TABACCO, La storia politica , cit., pp. 223, 239. Cfr. anche Giuseppe GALASSO, Potere e istituzioni in Italia. Dalla caduta dell'Impero Romano ad oggi, Torino, 1973, pp. $35 \mathrm{sgg}$., 113.

${ }^{27}$ E. SESTAN, Le origini delle signorie cit. da G. ChitTolini, Introduzione, cit., p. 20.

${ }^{28} \mathrm{G}$. CHITTOLINI, Introduzione, cit., p. 28.

${ }^{29}$ GiUlini, Memorie, p. 517.

${ }^{30}$ Paolo GRILLO, Milano in età comunale (1183-1276). Istituzioni, società, economia, Spoleto, 2001 , p. 665

${ }^{31}$ Giulini, Memorie, p. 519.
} 
La pace durò poco. L'anno successivo la pars populi "venne a trattare di eleggere un capo che avesse il titolo di anziano e signore" 32 . Dopo violenti scontri tra i componenti della "Credenza di S. Ambrogio" e la "Motta", culminati nell'assassinio di Azzolino Marcellino candidato di quest'ultima, Martino della Torre fu proclamato "anziano e signore del popolo di Milano" 33 . città $^{35}$.

Mandati in esilio tutti i "nobili" ${ }^{34}$ a lui nemici, divenne padrone della

Omettendo alcuni avvenimenti, che, pur attinenti al discorso, ci porterebbero lontano ${ }^{36}$, occorre soffermarsi sul titolo di "anziano e signore". Non si tratta più di una carica istituzionale in uso nel comune, esercitata su delega di questo in quanto detentore e fonte del potere nella sua collegialità, ma di una vera e propria magistratura di parte, che trae la sua legittimazione dalla sola pars populi in quel momento al potere, del quale di fatto è diventata depositaria $^{37}$, giacché la controparte dei "nobili" è in esilio.

Martino della Torre diventa l'effettivo e unico signore di Milano non con un colpo di stato, ma col libero consenso popolare, anche se limitato a una parte del "popolo". La carica di anziano della "Credenza di S. Ambrogio" non è limitata dalla durata annuale, come quella podestarile, ma è perpetua: è la nascita della signoria cittadina. Poco prima di morire, nel 1263, Martino ottiene che vengano trasmessi al fratello Filippo gli stessi suoi poteri che equivalgono a una podesteria perpetua del "popolo" ${ }^{38}$ : i della Torre ottengono la perpetua signoria mascherandola con un titolo meno odioso ${ }^{39}$.

Non solo è cambiata la sede del potere effettivo, non solo è cambiata la sua durata: anche il suo esercizio avviene al di fuori di ogni controllo. Un signore non è tenuto a render conto ad alcuno delle proprie scelte politiche.

${ }^{32}$ GiUlini, Memorie, p. 533.

${ }^{33}$ G. Fiamma, Manipulus Florum, cit., col 688; GiUlini, Memorie, p. 534.

${ }^{34}$ J. KOENIG, Il "popolo", cit., p. 122.

${ }^{35}$ G. FRANCESCHINI, La vita politica, cit., p. 294.

${ }^{36} \mathrm{La}$ lotta vittoriosa contro Ezzelino da Romano che, invitato dal "nobile" Guglielmo da Soresina, voleva impadronirsi di Milano nel 1259; l'interdetto di Urbano IV su Milano e la scomunica comminata a Martino della Torre, al fratello Filippo e altri cittadini in quanto fautori, allora, del re Manfredi e del ghibellino Uberto Pelavicino, al quale era stato attribuito il capitanato generale sulla città per cinque anni, dal 1259 al 1264 .

37،... fu nel sorgere di un potere autonomo, coesistente con il potere «comunale» e capace di autogoverno e di larga azione di governo nella città, ambiguo dunque istituzionalmente rispetto al «comune», non cioẹ rịducibile a puro strumento di concorrenza coị nobili per l'inserimento dẹ ceti popolari in organi già costituenti il «comune» e già operanti ufficialmente in nome della città" (G. TABACCO, Egemonie, cit., p. 281).

${ }^{38} \mathrm{Cfr}$. Luca DEMONTIS, Dal contado alla città e dalla città al contado: percorsi di potere dei della Torre tra politica comunale e interessi familiari. Un documento inedito del 1270 , in "Nuova Rivista Storica", anno LXXXIX, maggio-agosto 2005, fascicolo II, pp. 451-464, in particolare p. 458.

${ }^{39}$ GiUlini, Memorie, pp. 557-58. 
In teoria, secondo una caratteristica costante rilevata nelle signorie, egli non dovrebbe neppure assecondare la pars da cui è sostenuto e legittimato, giacché ha interesse a porsi super partes per facilitare la pacificazione sociale.

Il passaggio dalle istituzioni comunali a una forma di signoria avviene non solo in ambito concettuale, ma con segni e atteggiamenti concreti messi in atto dalla famiglia protagonista a Milano in questo periodo.

Nella condotta dei della Torre ci sono dei fatti rivelatori di un atteggiamento nuovo nell'esercizio del potere, dopo l'assunzione del titolo di anziano. Anzitutto c'è un riemergere della mentalità feudale da cui provenivano, riconoscibile nella riscoperta delle cerimonie d'investitura: nel 1266 Francesco della Torre, tornato a Milano dall'incontro con Carlo d'Angiò, dopo essere stato investito della contea di Venafro ${ }^{40}$, in una memorabile corte imbandita multos Milites militari balteo accinxit ${ }^{41}$. Di per sé una cerimonia del genere $^{42}$ sembrerebbe priva di rilevanza, ma vista in prospettiva prelude a un ritorno al "feudale", come in seguito si vedrà.

La pars populi, nonostante la convocazione delle assemblee e la promozione sociale di alcuni suoi membri, gradiva sempre meno il giogo torriano, che si faceva più duro col progredire degli anni e con l'aumentare delle tasse ${ }^{43}$. Forse intuiva che i capi erano meno attenti alle sue esigenze.

La preoccupazione dei Torriani in effetti pare concentrata nel trovare altrove, all'esterno del comune, una valida legittimazione all'esercizio del potere. Forse temono che un potere che proviene da una sola parte politica non sia riconosciuto in ambito internazionale: quindi è proprio in questo ambito che si sposta la loro richiesta di consensi.

Quando dalla città si allontana col proprio esercito Uberto Pelavicino, il cui capitanato generale scadeva l'11 novembre 1264 , i della Torre si liberano di una fastidiosa presenza. Egli infatti aveva tentato, senza successo, di minare alla base il potere di cui essi godevano, e aveva condizionato gli schieramenti politici di Milano.

La città sotto la piena signoria dei della Torre ottiene importanti successi in politica estera e ristabilisce l'antico orientamento guelfo temporaneamente abbandonato. L'alleanza con Carlo d'Angiò, al quale papa Clemente

\footnotetext{
${ }^{40}$ GiUlini, Memorie, p. 573.

${ }^{41}$ G. Fiamma, Manipulus Florum, cit., col. 694.

${ }^{42}$ Che sarà ripetuta ${ }_{a}$ per esempio, durante il passaggio a Milano di Margherita di Borgogna, futura sposa di Carlo d'Angiò (GIULINI, Memorie, p. 589).

${ }^{43}$ Il "popolo" era oberato da pesanti spese per sostenere i lavori pubblici e l'apparato bellico di Milano. Un fatto rivela la sua insofferenza: quando apprenderà la notizia della sconfitta dei della Torre ad opera di Ottone Visconti a Desio (1277) si ribellerà ai Torriani non tanto perché avesse un progetto politico alternativo, quanto perché aveva capito che la loro stella era tramontata. Un avvisaglia di scontento si era avuta nel 1259, quando molti membri delle categorie più elevate, appartenenti alla "Motta", avevano lasciato la pars populi per schierarsi con i "nobili", sospettando responsabilità dei Torriani nell'assassinio di Azzolino Marcellino, esponente di spicco della "Motta" rivale di Martino della Torre.
} 
IV aveva offerto la corona di re di Sicilia, porta i della Torre a riavvicinarsi gradualmente alla Sede Apostolica. Un membro della potente famiglia, Raimondo, vescovo di $\mathrm{Como}^{44}$, organizza alleanze politiche tra le città lombarde contro i ghibellini. Milano è a capo di una potente lega e i della Torre governano molte città direttamente, come podestà, o per mezzo di vicari. Napoleone (Napo) della Torre succede al cugino Filippo nel 1265: i membri della famiglia collaborano a mantenere un potere politico monocratico ed efficiente, proiettato nella grande politica internazionale.

Altri segni rivelano un nuovo modo di gestire il potere nei Torriani.

Nei trattati internazionali (con altre città, con altri signori e con Carlo d'Angiò) quando si parla di Milano non compare il solo nome della città, ma si nominano espressamente i Domini de la Turre ${ }^{45}$. Nel corso del 1272 il podestà di Milano Visconte Visconti, fratello di Tebaldo Visconti ${ }^{46}$, eletto papa il $1^{\circ}$ settembre $1271 \mathrm{col}$ nome di Gregorio X, fu il primo a prestare giuramento sul testo del sacramentum potestatis ${ }^{47}$ formulato da Napo della Torre e approvato dal Consiglio degli Ottocento il 7 gennaio: nel giuramento il podestà si impegnava a prestare obbedienza alla "Credenza" e a Napoleone della Torre quale anziano perpetuo del "popolo": un importante riconoscimento formale della signoria torriana ${ }^{48}$.

Un'altra innovazione si verifica quando i Torriani, negli ultimi anni del loro dominio signorile, attuano una grave violazione dell'assetto istituzionale del comune: il territorio viene riorganizzato secondo vecchi schemi feudali, abbandonati da almeno un cinquantennio, riesumati ora perché ritenuti più validi ed efficaci.

Il pieno controllo del contado da parte della città continuava ad essere un problema irrisolto. I "nobili" esiliati trovavano rifugio nei castelli e cercavano di attirare dalla loro parte gli abitanti di intere $z^{2} e^{49}$, promettendo benefici che il comune era restio a concedere, come l'uguaglianza di diritti con i cittadini.

Nella sua espansione verso il contado generalmente il comune si serviva di funzionari per il governo delle zone che voleva controllare. Ma in

\footnotetext{
${ }^{44}$ Nonostante le manovre del suo parentado per influenzare gli elettori del capitolo della cattedrale, non era andata a buon fine la sua çandidatura ad arcivescovo di Milano; la Sede Apostolica, per evitare concentrazioni di potere in mano ai della Torre a Milano, aveva preferito destinarlo alla sede di Como, e poi alla sede patriarcale di Aquileia, vasta, ricca e potente (Cfr. Maria Nadia CovINI, Della Torre Raimondo, in Dizionario Biografico degli Italiani, vol. XXXVII, Roma, 1989, p. 657).

${ }^{45}$ Gli atti del comune di Milano, cit., p. 474, doc. n. CDXXXII.

${ }^{46} \mathrm{Si}$ tratta della famiglia Visconti di Piacenza, non imparentata con l'arcivescovo di Milano Ottone Visconti.

${ }^{47}$ Gli atti del comune di Milano, cit., pp. 688-90, doc. n. DCXXXIX.

${ }^{48}$ Anna CASO, Della Torre Napoleone, in Dizionario Biografico degli Italiani, vol. XXXVII, Roma, 1989 , p. 623.

${ }^{49}$ P. GRILlo, comuni urbani, cit., p. 73.
} 
una situazione di instabilità, causata dalla presenza in loco della pars nobilium in esilio, questa prassi presentava dei rischi: poteva favorire il sorgere di forme di dominio personale ${ }^{50}$, proprio quelle che il comune voleva combattere.

I Torriani quindi portano avanti uno dei punti della politica della pars populi, cioè il controllo del contado; ma per realizzarlo non si servono di funzionari pubblici, bensì di membri del proprio casato ${ }^{51}$. Essi agiscono ponendosi al di sopra e al di fuori del comune: questa espansione nelle campagne appare come una conquista del territorio a livello familiare e privato. Contemporaneamente cambiano la vecchia suddivisione dei distretti, noti col nome di fagge ${ }^{52}$, per farne una nuova "su misura" per affermare ancora che la presa di possesso del contado avveniva non più a nome del comune ma del loro casato. Infatti vi insedieranno propri familiari o come domini loci $^{53}$ o come podestà rurali ${ }^{54}$ invece di mandare normali funzionari del comune.

Napoleone della Torre compie così una violazione sostanziale nei confronti della politica del "popolo". Non si preoccupa neppure delle reazioni di quest'ultimo, visto che esso non ha un programma alternativo ${ }^{55}$. Per mezzo di acquisti e concessioni i della Torre vengono in possesso di vaste aree del contado. Tra il 1272 e il 1276 Napoleone acquistò 92 appezzamenti di terra nel circondario di Turbigo ${ }^{56}$. Per tali acquisti si serviva, oltre che del patrimonio familiare, delle casse del comune. Poteva contare anche sulla partecipazione finanziaria di mercanti e banchieri interessati a investire nella proprietà fondiaria ${ }^{57}$. Nello stesso periodo Francesco della Torre acquisterà

\footnotetext{
${ }^{50}$ Viene sottolineato in modo particolare per la Lombardia orientale; cfr. François MENANT, Campagnes lombardes au Moyen Age. L'économie et la société rurales dans la région de Bergame, de Crémone et de Brescia du X.me au XIII.me siècle, Roma, 1993, "Bibliotheque de l'Ecole Française de Rome», 281, p. 524 ; P. GRILlO, comuni urbani, cit., p. 73.

${ }^{51}$ Cfr. L. Demontis, Dal contado alla città e dalla città al contado, cit., p. 459.

${ }^{52} \mathrm{La}$ città era divisa in tre grandi aree (fagge), ognuna delle quali corrispondeva a due porte civiche con relativo territorio e disponeva di propri consoli di giustizia (P. GRILLO, Comuni urbani, cit., p. 51).

${ }^{53}$ Francesco della Torre divenne dominus del comitato del Seprio (P. Grillo, Comuni urbani, cit., p. 75).

${ }^{54}$ Lombardo della Torre nel 1274 fu podestà di Albairate, zona di particolare interesse per la famiglia.

${ }^{55} \mathrm{G}$. CHỊTTOLINI, Città e contado nella tarda età comunale (a proposito di studi recenti), in "Nuova rivista storica", LIII (1969), p. 713.

${ }^{56}$ Di grande importanza strategica, perché controllava il corso del Naviglio (Flavia DE VITT La signoria dei della Torre in Turbigo, in "Bollettino storico bibliografico subalpino", LXXV (1977), pp. 636-37).

${ }^{57}$ F. DE VITT, La signoria, cit., p. 645.
} 
appezzamenti di terra e alcuni diritti di signoria nei dintorni di Trezzano sul Naviglio $^{58}$, con gli stessi intenti di Napoleone.

I della Torre vanno via via assumendo le prerogative dei signori contro cui il comune di Milano doveva affermare i suoi diritti. La funzione di "signore collettivo" 59 che la città assumeva nei confronti delle popolazioni del contado ora era gestita direttamente dalla famiglia della Torre, che in città deteneva il potere signorile istituzionalizzato con il sacramentum potestatis del $1272^{60}$.

Così si chiudeva, con percorso circolare, la marcia al potere dei Torriani: con un ritorno al contado e all'antica organizzazione del territorio che essi, conti di Valsassina, avevano per decenni abbandonato per aderire alle istituzioni comunali.

Come riconoscimento dall'alto del potere signorile della famiglia arriverà, nel 1274, il titolo di vicario imperiale per Milano concesso a Napoleone della Torre dal neo-eletto re dei Romani Rodolfo d'Asburgo ${ }^{61}$.

I della Torre, come si è visto, erano diventati signori di un potente stato territoriale e godevano del riconoscimento e dell'appoggio dell'imperatore e della Sede Apostolica. La loro fortuna finirà all'improvviso il 21 gennaio 1277, quando l'arcivescovo di Milano Ottone Visconti a capo della pars nobilium in esilio, dopo aver sconfitto a Desio l'esercito torriano, entrerà a Milano, acclamato signore dallo stesso "popolo" che aveva già dimenticato i della Torre. Gli scampati alla battaglia si rifugeranno ad Aquileia presso il patriarca Raimondo, loro potente congiunto. Ci sarà un loro ritorno a Milano nei primi anni del secolo XIV, ma non durerà a lungo $0^{62}$.

Duraturo si rivelerà, invece, il passaggio dal comune alla signoria.

\section{DALL'IMPERO DI BISANZIO AI GIUDICATI: I GIUDICI D'ARBOREA E L'ESPANSIONE IN SARDEGNA}

Per poter iniziare un discorso sull'origine dei giudicati sardi si deve investigare sull'arco di tempo compreso tra i primi decenni del secolo VI, quando Giustiniano riconquista l'Africa del nord e la Sardegna, e gli inizi del

\footnotetext{
${ }^{58} \mathrm{Cfr}$. Luca DEMONTIS, Il tentativo di signoria di Francesco della Torre in Trezzano sul Naviglio. I documenti della canonica di S. Ambrogio (gennaio 1276), "Aevum”, LXXXI (maggio-agosto 2007), pp. 485-522.

${ }^{59}$ G. SERGI, L'idea di Medioevo, in AA. VV., Storia medievale, Roma, 1998, p. 35.

${ }^{60} \mathrm{Cfr}$. Luca DemonTis, Dal contado alla città e dalla città al contado, cit., p. 458.

${ }^{61}$ Assieme al titolo arriverà una forza armata di 400 cavalieri, in risposta all'ambasceria che Napo della Torre gli aveva inviato per congratularsi; cfr. A. CASO, Della Torre, Napoleone, cit., pp. 621-625.

${ }^{62} \mathrm{Cfr}$. L. Demontis, Dal contado alla città e dalla città al contado, cit., p. 460.
} 
secolo XI, quando sull'isola intervengono con progetti di conquista le repubbliche di Pisa e di Genova ${ }^{63}$.

Data la scarsità di documentazione scritta del periodo gli studiosi sono costretti ad esprimersi al condizionale e a procedere per supposizioni. Piuttosto che dilungarmi su problemi generici, cercherò di cogliere gli elementi che servono alla mia ricerca.

Un dato significativo è questo: a partire dalla conquista dell'Africa da parte degli Arabi, nel 698, le forze del tema d'Africa con ogni probabilità si raccolsero in Sardegna e il titolo di patricius, che sin dal secolo VI era attribuito all'esarca d'Africa, fu assunto dal capo delle forze militari in Sardegna $^{64}$. La zecca imperiale fu trasferita per 25 anni a Caralis $^{65}$. L'isola, ancora parte integrante dell'Impero di Bisanzio,venne aggregata all'esarcato d'Italia e dipendeva da Ravenna. Poiché quest'ultima era lontana e impegnata a difendersi dai Longobardi, sperimentò una situazione di pre-isolamento.

In questo periodo hanno inizio alcune importanti trasformazioni sia a livello dell'organizzazione militare che in quello amministrativo, per adattare le forze presenti nell'isola alla situazione.

Il $d u x$ militare, che risiedeva a Forum Traiani (ora Fordongianus), e l'epistates della provincia chiamato sempre più spesso iudex Provinciae, che risiedeva a Caralis, hanno da fronteggiare le scorrerie dei Barbaricini dell'entroterra e dei Saraceni dal mare.

La magistratura di iudex non era nuova. Fin dall'età aurea dell'Impero Romano gli iudices avevano l'incarico dallo stato di garantire l'ordine e di amministrare la giustizia ${ }^{66}$. Gli iudices scomparvero insieme all'Impero d'Occidente, rimanendo in funzione solo in alcuni territori rimasti legati a Bisanzio: la loro funzione nei regni romano-barbarici, e in seguito nell'Impero carolingio, venne ricoperta dal personale nominato dal sovrano, che esercitava in suo nome l'autorità pubblica: i conti.

Al sistema dell'esercito mobile dei comitatenses $^{67}$, impotente ad affrontare il pericolo in zone tra loro lontanissime, si sostituisce l'organizzazione dei soldati limitanei ${ }^{68}$, reclutati fra $\mathrm{i}$ possidenti di terre marginali (possessores), che presto assumono una certa influenza in ogni

${ }^{63}$ Il fatto storico che ne causò l'intervento armato fụ il tentativo, non riuscito, da parte dị Mujahid principe di Denia, di stabilire possedimenti stabili in Sardegna nel 1015-16. I Saraceni volevano basi in prossimità delle coste della penisola italiana da loro ripetutamente saccheggiate. Per evitare questo rischio Pisa e Genova, dopo averne allontanato i Saraceni, iniziarono la loro conquista dell'isola a danno dei giudicati, strutture di governo ormai saldamente affermate.

${ }^{64}$ Sarebbe identificabile nella sua persona il "batrica di Sardinia che governa le isole del mare" di fonte araba (CASUlA, Dizionario, v. Esarcato d'Africa, p. 581).

${ }^{65}$ CASUla, Dizionario, v. zecca, p. 1595.

${ }^{66}$ San Paolo stesso, in alcune sue lettere, fa riferimento all'autorità di tali magistrati romani; essi, dice, non portano la spada invano e ad essi è opportuno sottomettersi (Rom. 13,4; Tit. 3,1) (J.FLORI, La cavalleria medievale, Bologna, 2002, p. 12).

${ }^{67}$ Posti sotto il comando di Comites rei militaris (Giuseppe CORRADI, v. Bizantino, Impero, Grande Dizionario Enciclopedico UTET, III, Torino, 1967, p. 153).

${ }^{68} \mathrm{Di}$ regola stanziati lungo il confine (limes). 
settore della vita pubblica ${ }^{69}$. Tra le famiglie più importanti emergono i Lacon e i Gunale, che in seguito si fonderanno in un unico clan familiare da cui avranno origine le dinastie dei futuri giudicati ${ }^{70}$.

A causa delle frequenti incursioni saracene dal 705-706 in poi, scatta in Sardegna il sistema bizantino dell'autocrator ${ }^{71}$. Infatti subito dopo non si parla più del dux di Forum Traiani ma di un'unica autorità dell'isola: lo iudex Provinciae $^{72}$. L'isola, benché ancora dipendente da Bisanzio e soggetta ad accogliere $i$ rescritti che fossero pervenuti tramite gli apocrisiari ${ }^{73}$ dell'imperatore, guadagna una "autonomia di fatto" 74 e tra i secoli VIII e XI si trova "sciolta da ogni effettiva soggezione verso il lontano Impero d'oriente" 75 . È un cammino che procede per gradi.

Già prima che l'occupazione araba della Sicilia, iniziata nell'827, porti a un maggiore isolamento della Sardegna, un avvenimento significativo rivela che nell' isola c'è già la coscienza del suo inevitabile distacco dall'orbita di Bisanzio. Nell'815 il giudice provinciale di Cagliari invia una delegazione alla corte di Ludovico il Pio a Francoforte (Traiectum) per pagare un tributo (dona) ai Franchi in cambio di aiuti per contrastare gli assalti provenienti dal Maghreb $^{76}$.

Una decisione del genere rivela che probabilmente i governanti dell'isola hanno già fatto analoghe richieste d'aiuto a Bisanzio senza ottenere risposta. Ciò non vuol dire però che quel tributo assuma ulteriori significati: quando i Sardi pensano all'Impero hanno sempre in mente Bisanzio. Forse nel gesto c'è una più acuta percezione che l'isola è una zona di confine, una "terra di mezzo" abbandonata di fatto a se stessa, e quindi spinta dalle circostanze a prendere iniziative al di fuori delle regole.

I Sardi non ottengono aiuti concreti dai Franchi e dovranno continuare a respingere da soli gli assalti dei Saraceni; ma il conte Bonifacio, prefetto della Corsica, quando nell'828 approda in Sardegna la chiama insula amicorum $^{77}$. Questi fatti sono la prima testimonianza documentata della percezione in senso politico che i Sardi hanno dell'occidente, in cui sono

\footnotetext{
${ }^{69}$ Casula, Dizionario, v. Sardegna Bizantina, p. 1595.

${ }^{70}$ Casula, Dizionario, v. Lacon-Gunale, p. 820.

${ }^{71}$ Ossia di un unico comandante con funzioni sia civili che militari, più agile nelle funzioni di governo e più adatto alle situazioni d'emergenza. Equivale al termine romano di dittatore; cfr. Grande Dizionario Enciclopedico, II, Torino, 1968, v. autocrazia, p. 466.

${ }^{72}$ Per l'evoluzione delle magistrature bizantine cfr. G. TABACCO, Egemonie, cit., pp. 98-101.

${ }^{73}$ Erano i funzionari bizantini incaricati di portare le direttive dell'imperatore alle province, cfr. CaSUla, Dizionario, v. Apocrisiario, p. 65.

${ }^{74}$ SolmI, Studi, introd. p. VIII.

${ }^{75}$ Solmi, Studi, p. 3.

${ }^{76}$ CASUla, Dizionario, v. Sardegna bizantina, p. 1597. Nell'846 i Saraceni saccheggeranno la stessa Roma.

${ }^{77}$ Solmi, Studi, p. 12.
} 
geograficamente inseriti, nonostante i legami con Bisanzio, che restano ben saldi, invece, dal punto di vista culturale ${ }^{98}$.

Nell'isola continua ad essere usato ancora per molto tempo il "bisante" come moneta e unità di conto, fino a quando gli intensificati rapporti con le città della penisola apriranno le porte a nuove monete ${ }^{79}$. In ciò essa si differenzia da una costante tendenza dei comuni più forti a coniare moneta propria come segno di indipendenza dal potere imperiale ${ }^{80}$.

Intanto l'isola attira l'attenzione di vari papi per i molteplici problemi legati alla sua decadenza. Le lettere papali sono fonte di preziose notizie. Di spiccato interesse è l'intervento di papa Nicolò I nell'864: i suoi delegati si rivolgono non a una sola autorità isolana ma ai giudici, per condannare la loro abitudine di contrarre nozze tra consanguinei. Il rimprovero si ripete, sempre diretto agli iudices, al plurale, in una lettera di papa Giovanni VIII nell' $873^{81}$.

In quegli anni evidentemente ci sono nell'isola "autorità sovrane, presupponenti la formazione giuridica di più Stati locali autonomi - i regni giudicali o «giudicati» -, in conseguenza della fine di fatto della dipendenza dell'isola dall'Impero di Bisanzio" 82 .

Quindi si ipotizza che dall'originario e unico iudex o governatore, che delegava il solo potere militare nelle zone periferiche a un loci servator, scelto tra i familiari di sua fiducia ${ }^{83}$, siano nati altri centri di potere in ogni distretto, quando il loci servator allargava le sue competenze assumendo il governo del territorio: ciò sarebbe avvenuto o per "usurpazione" o per riconoscimento spontaneo della famiglia da cui proveniva ${ }^{84}$.

L'origine dei giudicati risalirebbe dunque all'antico istituto giustinianeo del loci servator, diventato di fatto, e poi anche di diritto, giudice ${ }^{85}$.

Anche in Sardegna forse c'è stata una fase di sperimentazione della gestione del potere, finché dei molti loci servatores se ne affermarono quattro, numero corrispondente a quello dei giudicati storici. È riferibile quindi a

\footnotetext{
${ }^{78}$ Questi legami culturali sono stati assunti da alcuni studiosi come prova di una dipendenza politico-amministrativa che si sarebbe mantenuta fino alla fine del periodo considerato (secoli VIXI). Ne resta traccia nell'onomastica di luoghi, chiese e santi, e negli influssi lasciati dai monaci provenienti dall'oriente.

${ }^{79} \mathrm{Nel}$ periodo giudicale in Sardegna, dal secolo X al XIV, si usano solo monete d'importazione (CASUlA, Dizionario, v. monetazione giudicale, p. 982).

${ }^{80}$ A Milano la zecca cittadina nel 1273 cominciò a coniare l'ambrogino d'oro. Il conio dei comuni guelfi sottolinea tale indipendenza, mentre signori e comuni ghibellini mettono in risalto l'autonomia dell'istituzione comunale come concessione dell'imperatore, di cui riconoscono la sovranità incidendone il nome. In Sardegna "la prima vera zecca, attestata con la coniazione del gran tornese d'argento, fu nel 1289 quella di Villa di Chiesa (ora Iglesias), nello stato signorile dei Gherardesca ugoliniani", da cui uscirono nel 1302 i grossetti o aquilini, recanti sul dritto una croce e la legenda: FACTA IN VILLA ECCLESIE P(ER) COM(UN)I PISANO; sul rovescio l'aquila imperiale e la scritta celebrativa FEDERIC(US) IN(PER)ATOR (CASULA, Dizionario, v. zecca, p. $1920)$.

${ }^{81}$ CASUla, Dizionario, v. Sardegna bizantina, p. 1598.

${ }^{82}$ Ibid.

${ }^{83}$ Alberto Boscolo, La Sardegna dai giudicati all'età comunale, in AA. VV., Breve storia della Sardegna, Torino, 1965, p. 95.

${ }^{84}$ A. Solmi, Studi, pp. 27-28.

${ }^{85}$ Il nome si è affermato per influsso della Chiesa, giacché negli atti pontifici "Giudice” o "Arconte" era il titolo dato ai capi supremi del governo nell'isola (A. SolMI, Studi, p. 19).
} 
queste circostanze e a questo periodo la nascita delle prime forme di potere signorile $^{86}$. Il giudicato, con la sua semplice struttura, era adatto per un governo a forte autorità centrale.

L'eredità bizantina è rimasta anche nell'articolazione del governo territoriale. Il contado è suddiviso in distretti o curatorias o partes, a capo dei quali il giudice prepone degli ufficiali, i curatores, scelti tra le persone di sua fiducia.

Mentre in "Lombardia" e in Toscana le egemonie territoriali efficienti gravitavano normalmente sulle città ${ }^{87}$, in Sardegna erano molto più legate a dinastie regie o a signorie rurali: l'organizzazione dei distretti, per il giudicato d'Arborea, sembra riproporre in piccolo quella della corte giudicale. Leggendo la Carta de Logu di Eleonora d'Arborea, di cui si parlerà oltre, si ha l'impressione di una realtà rurale molto ben organizzata fin nei minimi particolari $^{88}$, saldamente legata alla realtà urbana del capoluogo. Per la città di Milano, al contrario, il controllo del contado costituiva una spina nel fianco: i della Torre furono costretti a rimettere in funzione una organizzazione di tipo feudale per far fronte all'insicurezza che regnava nelle campagne e al pericolo che vi nascessero centri di potere autonomo. L'estensione territoriale e le risorse di ogni distretto del giudicato erano talmente modeste che nessun curatore poteva ragionevolmente fare progetti concreti di autonomia. Non vi erano neppure altri centri di potere autonomi, come invece esistevano ai confini del territorio del comuni di Milano. Quanto all'organizzazione definita "feudale", essa era assolutamente sconosciuta nel giudicato d'Arborea: venne introdotta in parte quando alcune famiglie pisane fondarono dei poteri signorili ${ }^{89}$, e in modo generalizzato quando gli Aragonesi conquistarono l'isola.

In Sardegna non c'è stata una città in contrasto col contado, una realtà cittadina proiettata alla conquista degli spazi rurali, non solo perché il fenomeno della genesi della città non si è pienamente verificato, ma perché le due realtà, anziché essere in contrasto, si fondono tra loro. Una prova la troviamo nella legislazione: non ne esiste una riservata ai cittadini e un'altra riservata ai rustici. Era questo contrasto che nella "Lombardia" opponeva città e campagna come due realtà in antagonismo, per cui era naturale che la città andasse alla conquista del contado. Perché grandi zone del contado lombardo da un giorno all'altro potevano abbandonare il legame di dipendenza con la città e passare dalla parte dei "nobili" in esilio? Perché questi ultimi promettevano, in caso di vittoria, di togliere ai rustici i pesi a cui li sottopone-

86“Nell'isola, frazionata in un numero imprecisato di aree d'influenza, ma certamente superiore alle quattro unità che daranno vita ai rispettivi regni giudicali, esisteva un potere signorile" (Giuseppe MELONI, L'origine dei giudicati, in Storia della Sardegna, 2 Dal Tardo Impero Romano al 1350 a c. di Manlio BrigagliA, Attilio MASTINO, Gian Giacomo ORTU, Roma-Bari, 2002, p. 27).

${ }^{87}$ G. TABACCO, Egemonie, cit., p. 315.

${ }^{88}$ Avvalorando l'ipotesi che alla sua base ci sia un codice rurale del padre Mariano IV.

${ }^{89}$ La famiglia Visconti (alla quale apparteneva il Nino che Dante ricorda al v. 53 del canto VIII del Purgatorio) aveva diritti sulla Gatlura e una parte del Giudiçato di Cagliari; i Donoratico si radicarono nella zona di Villa di Chiesa, dando impulso all'attività estrattiva. 
va il comuni cittadino, e di renderli giuridicamente uguali agli abitanti della città.

Questo rischio non c'era nel giudicato d'Arborea, dove le leggi, prima consuetudinarie e poi codificate, erano uguali per tutti, sia che abitassero in città, sia che abitassero in campagna.

Il giudice esercita la piena autorità (rennare, potestare, imperare) derivandola "da doppia fonte: l'eredità, che legittima il potere, e l'elezione, che manifesta l'autorità dei sudditi e principalmente dei maiorales $^{90}$ : l'una $\mathrm{e}$ l'altra concorrono insieme a costituire la sovranità" 91

Tra i maiorales c'è un sostanziale equilibrio che torna avantaggio del giudice in carica, nel senso che nessuna di queste famiglie è così potente da poterlo scacciare dal trono.

Nelle motivazioni che giustificano la presa del potere da parte dei giudici sono presenti due componenti: quella elettiva, propria di un potere che deve trovare l'assenso del popolo o almeno della parte più evoluta di esso, e quella ereditaria. Mentre la prima componente c'è nelle due realtà che stiamo esaminando, la seconda, quella ereditaria, si è sviluppata e imposta in Sardegna parallelamente allo sviluppo dei giudicati, ma è assente nei comuni italiani: comincia ad esistere solo quando si sviluppa la signoria, come si è visto per la famiglia della Torre a Milano.

Si potrebbe cercare quale delle componenti - ereditaria o elettiva abbia la preminenza all'interno dei due sistemi o in altri termini, quale delle due sia sentita come la vera fonte del potere.

Nella storia del comune c'è una certa evoluzione in questa percezione.

A Milano nel secolo XI, com'è noto, al governo del vescovo che esercita anche il potere temporale si affiancano elementi che provengono dalla nobiltà feudale inurbata e dai ceti più evoluti del "popolo" 92

Quando questi ultimi formano una matura classe politica e la cittàcomune lotta non solo per la sua autonomia dall'Impero, ma anche per controllare i privilegi dei signori nel contado, non c'è dubbio che il potere sia percepito come proveniente dal comune in quanto istituzione creata dai cittadini, davanti al quale sono responsabili e giurano fedeltà tutti coloro che vi ricoprono delle cariche.

Finché dura l'istituzione comunale (mi riferisco sempre a Milano, ma il paragone potrebbe essere valido anche per altri comuni), l'investitura del

\footnotetext{
${ }^{90} \mathrm{Nei}$ Giudicati essi erano "i liberi maggiorenti, i li (b)eros mannos, compresi i re, discendenti del vecchio ceto dirigente tardo imperiale e bizantino, chiamati anche donnos, proprietari, nel «pegugiare» dei villaggi, di terras clausas con coloni e servi" (CASULA, Dizionario, v. maiorale, p. 893). I re distinguevano il patrimonio privato (pegugiare) da quello statale.

${ }^{91}$ Solmi, Studi, p. 70. Il rapporto tra il sovrano e il suo popolo è regolato dal banno-consenso: al re viene concesso di governare "in cambio del rispetto delle prerogative popolari espresse nel giuramento all'atto della intronizzazione". La rottura di questo patto poteva indurre al tirannicidio: nell'Arborea ciò avvenne nel 1307 con l'uccisione di Giovanni Chiano e nel 1383 con quella di Ugone III (CASULA, Dizionario, v. banno, p. 149).

${ }^{92}$ In questa "pluralità di ceti fondatori sta la prima grande specificità del fatto comunale italiano" (Enrico ARTIFONI, Città e comuni, in AA. VV., Storia medievale, Roma,1998, p. 371).
} 
potere proviene dalla volontà dei cittadini ${ }^{93}$ : fu il "popolo" a chiamare Pagano della Torre per suo podestà e difensore. A un potere che discendeva dall'alto, corredato nel suo conferimento da cerimonie religiose, se ne sostituisce un altro che sale dal basso, ma che ha una legittimazione altrettanto forte e addirittura sacrale, se è vero che vox populi, vox Dei $i^{94}$.

Nel "popolo" questa percezione è alimentata da una graduale maturazione politica che avviene con il confronto e la partecipazione alle assemblee, con la cura e la difesa degli spazi pubblici, valori contrastati dall'aristocrazia signorile, che tende a chiudersi nel suo isolamento, non solo simbolico, all'interno dei castelli del contado.

In seguito, come si è visto nella storia della famiglia della Torre, il potere, anziché essere emanazione dell'intera comunità, viene accaparrato dalla pars che prende il sopravvento nella gestione del potere: questa elegge a suoi rappresentanti delle persone che provengono spesso dal vecchio ceto signorile $^{95}$, che a loro volta tendono a ottenere un riconoscimento perpetuo della magistratura che ricoprono all'interno del comune.

Mentre il "popolo" continua a credersi l'unico depositario-detentore del potere, il suo rappresentante eletto cerca delle conferme esterne, come il titolo di vicario imperiale, e impone, strappando il consenso al "popolo", che i poteri di cui gode siano trasmessi ai suoi eredi.

È nata la signoria. Questo fatto implica un mutamento di pensiero non solo sulle modalità di esercizio del potere, ma sul concetto e sull'origine del potere stesso: da elettivo, collegiale e limitato nel tempo, esso diventa monocratico, trasmissibile in successione (quindi senza limiti di durata), e sostanzialmente separato dalla fonte da cui proveniva prima.

Dal sistema comunale, nato dal contrasto con l'Impero, nasce un nuovo modo di gestire il potere, non più elettivo, ma ereditario, la signoria, che sente la necessità di avere una consacrazione ufficiale da un'autorità superiore, in particolare da quella imperiale.

Nel sistema del giudicato le due componenti, ereditaria ed elettiva, sono sempre esistite e andate di pari passo: difficile ipotizzare quanto conti l'una e quanto l'altra nella designazione della persona del giudice.

Sin dal primo cedimento della presenza politica bizantina, infatti, la Sardegna non deve affatto lottare come i comuni per ottenere l'autonomia dall'autorità superiore: in una prospettiva di autogoverno viene a trovarsi spontaneamente, senza esservi preparata, a partire dall'occupazione araba dell'Africa. La scelta dei governatori civili, secondo le disposizioni imperiali,

\footnotetext{
${ }^{93}$ Sono i rappresentanti dei cittadini riuniti in assemblea che eleggono alle magistrature comunali i loro delegati: nel periodo del comune "consolare" appartengono alla nobiltà cittadina; in quello "podestarile" figurano già magistrati del "popolo"; nel periodo del comune di "popolo", infine, le magistrature sono in un primo momento equamente divise, poi gestite solo dalla pars populi al potere.

${ }^{94} \mathrm{Per}$ quanto riguarda i Milanesi, in particolare, non può sfuggire l'analogia con l'episodio che vede Ambrogio acclamato vescovo.

${ }^{95}$ Uno dei più vecchi rappresentanti del "popolo" appartenente alla nobiltà nella lotta contro i privilegi dẹi "nobili" è Lanzone, nobilis et capitaneus altus, nell”XI secolo (G. TABACCO, Egemonie, cit., pp. 228-29).
} 
era affidata ai vescovi e ai notabili delle province ${ }^{96}$, col proposito di rendere istituzionale il collegamento fra il potere del principe e i quadri tradizionali della società romano-italica ${ }^{97}$. Ciò sembrerebbe conferire alla nascita dei giudicati una componente prevalentemente elettiva. Ma molto presto l'affermazione al potere di un unico clan familiare, i Lacon-Gunale, aggiunge la componente ereditaria: i quattro giudicati appaiono già come delle signorie, in cui però convivono ambedue le componenti. La coscienza del potere regale da trasmettere agli eredi spingerà i giudici a seguire un costume molto in voga a partire dal X-XI secolo nei regni e nelle signorie d'Europa: inviare i propri figli alla corte di un potente parente affinché vi fossero «nutriti», vale a dire educati come scudieri, servendo «in armi» fino alla loro vestizione da parte di quello stesso signore ${ }^{98}$.

I giudici avevano adottato anche una consuetudine riscontrabile nel governo centrale di Bisanzio. Il basileus, che all'inizio doveva essere eletto ed approvato per acclamazione dal senato, dall'esercito e dal popolo, e poi incoronato dal patriarca di Costantinopoli, in seguito designava in anticipo il proprio successore, adottandolo e associandolo al governo, senza che questa prassi portasse mai a un diritto ufficiale di successione al trono ${ }^{99}$.

Anche il giudice in carica ricorreva a questa prassi, sempre con l'approvazione dell'assemblea dei maiorales: una volta ratificata la "associazione al trono" doveva risultare quasi naturale riconoscere nella persona scelta dal giudice il suo successore. Sembrerebbe che il governo dei quattro giudicati sia regolato da un sistema perfettamente equilibrato. In effetti si tratta di signorie territoriali che nonostante le inevitabili guerre si mantengono in sostanziale equilibrio fra loro.

Dopo l'intervento di Pisa e Genova, che riporta l'isola nella sfera politica occidentale, la Sardegna compie il suo graduale avvicinamento all'occidente sia in campo religioso che politico.

Si è parlato del radicamento di tradizioni religiose della Chiesa greca, alcune delle quali perdurano anche oltre lo scisma del $1054^{100}$. In realtà la Chiesa Sarda gode per molto tempo, come il potere politico, di una forte indipendenza rispetto a quella di Bisanzio, in quanto autocefala, perché

\footnotetext{
${ }^{96}$ Spesso i funzionari bizantini, nonostante le proibizioni, conseguivano l'ufficio col pagamento di una grossa somma (suffragium) e si rifacevano con gravose tasse sui possessores, sul clero e sui poveri (SOLMI, Studi, p. 8).

${ }^{97}$ G. TABACCO, Egemonie, cit., p. 91.

${ }^{98}$ Questo costume era già in uso presșo i Longobardi, come ci racconta Paolo Diacono: i figli dei re longobardi non venivano ammessi alla tavola del padre se prima non erano stati giudicati degni di ricevere le armi per mano di un altro re in un'altra corte (J. Flori, La cavalleria, cit., p. 24).

${ }^{99}$ Durante l'Impero Bizantino și succedettero in dieci dinastie un centinaio di imperatori: 39 per filiazione dinastica, 4 per adozione, 4 senza vincolo dinastico, ma per regolare elezione, 9 per matrimonio e una trentina per usurpazione (G. CORRADI, Grande Dizionario Enciclopedico, cit., v. Bizantino, Impero, p. 153).

${ }^{100}$ Per esempio, l'uso di portare la barba lunga per gli ecclesiastici; la benedizione delle case per l'Epifania; la venerazione degli angeli e il culto dell' 'imperatore Costantino, iniziato fra gli Ariani. Papa Gregorio VII sostitui al rituale greco quello romano con l'aiuto dei Vittorini (A. BoSCOLO Dai Vandali ai Bizantini, in AA. VV., La società in Sardegna nei secoli, Torino, 1967 (rist. 1972), pp. 103-04).
} 
l'arcivescovo di Cagliari, primate metropolita, viene eletto da una sinodo di vescovi locali a loro volta nominati dal metropolita ${ }^{101}$.

Nei giudici sardi si fa strada la preoccupazione di legittimare il potere, detenuto di fatto e di diritto per il distacco da Bisanzio, presso le supreme autorità con cui ora hanno a che fare: il papa e l'imperatore del Sacro Romano Impero Germanico. A partire dalla metà dell'XI secolo cercano di agevolare l'insediamento di monaci nell'isola con donazioni di chiese, terre, servi, bestiame e, per i Benedettini Vittorini di Marsiglia, di saline ${ }^{102}$. Le donazioni sono fatte talvolta alle "opere" ${ }^{103}$ di chiese pisane e genovesi.

Il Giudicato d'Arborea agli inizi del XII secolo è ancora sostanzialmente indenne da questa penetrazione, che viene guardata, soprattutto quella pisana, con una certa preoccupazione. La consapevolezza della fragilità del proprio regno di fronte a questa invadenza straniera e la rivalità dichiarata tra Pisa e Genova spingono Barisone I giudice d'Arborea a concepire un disegno ambizioso: ottenere dall'imperatore Federico I il titolo di rex Sardiniae, un riconoscimento che avrebbe fornito la base giuridica per l'espansione in tutta l'isola $^{104}$. Sardegna ${ }^{105}$

Per la prima volta il potere laico imperiale creava questo titolo per la

Barisone chiese appoggio a Genova, che gli prestò la somma di 4000 marchi richiesta da Federico I per l'investitura. La corona nominale di re di Sardegna, ottenuta nell'agosto del 1164 a Pavia, non solo gli costò troppo cara $^{106}$, ma non sortì alcun effetto di espansione territoriale nell'isola. Per di più nel 1165 l'imperatore con gran disinvoltura infeudò Pisa di tutta la Sardegna, vanificando la concessione fatta a Barisone. Restava un titolo esclusivamente nominale.

Un riconoscimento alla preminenza del giudicato d'Arborea sugli altri verrà un secolo dopo: papa Clemente IV nominerà Mariano di Bas vicario della Sede Apostolica in Sardegna nel 1265, titolo che gli darà qualche

VII ${ }^{101}$ Consuetudine accettata anche dai papi, che finì col Dictatus papae del 1075 di Gregorio

${ }^{102}$ Quando i Giudici d'Arborea richiesero monaci dalla penisola avevano l'intenzione di usarli nelle proprie cancellerie e per diffondere la cultura.

${ }^{103}$ Associazioni di laici che amministravano i beni della chiesa.

${ }^{104}$ Sarebbe stata la corte imperiale a inviare nel 1158 due legati nell'isola e a proporre per prima l'investitura di Barisone a re di Sardegna (G. G. ORTU, I giudicati, cit., p. 39).

${ }^{105}$ CASULA, Dizionario, v. Barisone I re d'Arborea, pp. 159-69. Il titolo verrà conferito da Federico II al figlio Enzo nei 1238 a Cremona (il principe aveva sposato e poi abbandonato Adelasia, titolare del Giudicato di Torres). Qualche decennio dopo, in un periodo meno felice per l'Impero, sarà la Sede Apostolica a rivendicare il diritto di concedere in feudo la Sardegna nel 1267 con Clemente IV, progetto che realizzerà Bonifacio VIII il 6 aprile del 1297 investendo Giacomo II d'Aragona "vessillario, ammiraglio e capitano generale della Chiesa" del titolo di Rex Sardiniae et Corsicae (SolmI, Studi, pp. 297-98) in cambio della rinuncia alla Sicilia, sulla quale il papa rivendicava gli stessi diritti.

${ }^{106}$ Poiché non riuscì a sdebitarsi coi Genovesi dovette consegnare in pegno la sua persona fino al 1172 oltre a numerosi ostaggi e beni, cfr. Codice Diplomatico della Repubblica di Genova dal MCLXIII al MCLXXXX, a c. di Cesare IMPERIALE DI SANT'ANGELO, V. II, Roma, 1938, docc. n. 34 (1168, ottobre 23), n. 35 (1168), n. 36 (1168), n. 37 (1168). 
vantaggio nella controversia ereditaria con i Capraia ${ }^{107}$, ma non per conseguire il titolo ben più altisonante di re di Sardegna, per il quale il papa preferirà altri candidati.

La tendenza a trasformare il giudicato in regno per mezzo dell'investitura papale, e quindi ad avere un riconoscimento internazionale in ambito occidentale, viene vanificata dalla presenza di Pisa e Genova, che espandendosi nell'isola la considerano un prolungamento del proprio contado $^{108}$, dove le famiglie delle due città si creano, a loro volta, vaste zone di potere signorile.

Per i Pisani la Sardegna, oltre a costituire un importante sbocco per la creazione di signorie rurali e lo sfruttamento minerario dell'Iglesiente, era un utilissimo punto di approdo e approvvigionamento delle navi destinate agli scambi commerciali con la Tunisia (emirato di Ifriqya), dove i Pisani godevano di particolari privilegi ${ }^{109}$.

L'isola era altrettanto importante per i Genovesi non solo per stabilirvi basi d'appoggio, ma per sfruttarne le risorse, tra le quali avrà grande importanza il corallo ${ }^{110}$.

\section{CONCLUSIONI}

Ho cercato di descrivere il percorso verso il potere di due realtà derivanti dalla crisi dell'istituzione imperiale, sia del Sacro Romano Impero Germanico nella penisola italiana, sia dell'Impero di Bisanzio nell'isola di Sardegna.

Paradossalmente il potere acquisito "dal basso" sente la necessità di una legittimazione "dall'alto", da quell'istituzione imperiale da cui si erano prese le distanze.

Mentre per il comune di Milano, ricco e potente, il conferimento del vicariato imperiale coronerà una situazione di effettivo potere dei della Torre esteso alla Lombardia, e costituirà un riconoscimento del nuovo regime signorile, per i signori di Arborea ${ }^{111}$, poveri di mezzi e stretti fra le due potenze di Genova e Pisa, l'investitura a re di Sardegna non cambierà nulla. Occorrerà attendere gli ultimi decenni del secolo XIV per vedere il Giudicato d'Arborea in piena espansione territoriale, quando la reggente Eleonora,

${ }^{107}$ G. G. ORTU, I giudicati, cit., pp. 51-52.

${ }^{108}$ G. G. ORTU, I giudicati, cit., p. 42.

${ }^{109}$ Archivio del Comune di Pisa, Atti Pubblici, docc. n. 108, 1204 maggio 17; n. 117 (1211 settembre 9); n. 157 (1264 agosto 9). Cfr. anche Marco TANGHERONI, Il'Mediterraneo bassomedievale, in AA. VV., Storia Medievale, Roma, 1998, pp. 469, 484 e 490. Spesso i mercanti fiorentini si dichiaravano "pisani" per godere degli stessi vantaggiosi privilegi.

${ }^{110} \mathrm{Cfr}$. Roser SALICRÙ I LluCH, Una llutta per un mercat: Catalans, Algueresos $i$ Genovesos i el corall de Sardenya a la primiera del segle XVI, in Maria Teresa FERRER I MALLOL, Josefina MUTGE I VIVES, Manuel SÁNCHEZ MARTINEZ, La corona catalanoaragonesa $i$ el seu entorn mediterranei a la baixa edat mitiana, Barcelona, 2005, pp. 309-362.

${ }^{111}$ Che hanno il nome di Lacon-Serra, e che dal 1194 assumeranno quello di Bas (Bas-Serra) quando Ugone I avrà il titolo di visconte di Bas (CASUla, Dizionario, v. Bas, p. 166). 
juyghissa (giudicessa) di fatto e non di diritto, arriverà a conquistare, anche se per poco, quasi tutta la Sardegna ${ }^{112}$. L'investitura del titolo di re segna comunque il definitivo passaggio dell'isola nell'orbita occidentale: per i Sardi 1' "Impero" ormai è in Occidente.

La vera e duratura conquista che il Giudicato d'Arborea mise in atto nell'isola intera, sempre ad opera di Eleonora, fu di natura giuridica. Sotto il suo regno fu completato e promulgato il codice di leggi chiamato Carta de $\log u$, la cui validità fu riconosciuta anche dagli occupanti ispanici e mantenuta in vigore per secoli ${ }^{113}$.

Data di ricezione dell'articolo: febbraio 2007.

Data di accettazione e versione finale: dicembre 2007.

${ }^{112}$ Resteranno fuori solo le città di Alghero e Cagliari.

${ }^{113}$ Iniziata dal padre Mariano IV sulla base delle consuetudini, la Carta si giovò degli statuti sassaresi e di quelli di Castelgenovese, del Breve di Villa di Chiesa, delle leggi pisane e genovesi; ebbe la meglio, come diffusione, sull'analoga Carta di Cagliari, redatta in latino, perché sí esprimeva in volgare ed era comprensibile a tutti (Cfr. SolMI, Studi, pp. 271 sgg.). 